\title{
Análisis de la concepción de docentes y estudiantes sobre el juego como recurso didáctico para el aprendizaje: experiencia en la educación primaria Analysis on Teachers' and Students' Perceptions on Game as a Didactic Resource for Learning: Experience in Elementary Education
}

\author{
Mónica Patricia Melo Herrera \\ Universidad Distrital Francisco José de Caldas die UD, Colombia \\ ud2000admon@yahoo.com
}

\section{RESUMEN}

El juego ha sido concebido socialmente como una actividad con la cual se gasta energía y se pasa el tiempo, sin tener en cuenta su carácter potenciador del aprendizaje. El presente artículo tiene como objetivo identificar y reflexionar sobre la concepción que docentes y estudiantes tienen respecto al juego como recurso didáctico en los procesos de enseñanza y aprendizaje. Se tienen como base los resultados de una encuesta utilizada en el marco de un trabajo de investigación de corte cualitativo sobre el desarrollo de habilidades comunicativas en un colegio de la ciudad de Bogotá. Para su estudio se diseñaron categorías de análisis emergentes basadas en los objetivos de la investigación. Estos resultados mostraron que docentes y estudiantes valoran el uso del juego como actividad para promover el aprendizaje, pero no se emplea debido a la percepción que se tiene del mismo. Insumos de este trabajo se han empleado para la investigación del nivel doctoral sobre interculturalidad en la escuela.

Palabras clave: percepción, juegos, recursos didácticos, enseñanza, aprendizaje.

\begin{abstract}
The game has been conceived socially as an activity with which energy and time are spent without taking into account its enhancer character of learning. This article aims to identify and reflect on the conception that teachers and students have regarding the game as a didactic resource in the teaching and learning processes. It is based on the results of a survey carried out in the framework of a qualitative research work on the development of communicative skills in a school in the city of Bogotá. For their study, emerging analysis categories were designed based on the research objectives. These results showed that both teachers and students value the use of the game as an activity to promote learning, but they do not use it due to the general perception of it. Inputs of this work have been used for research work at the doctoral level on interculturality in the school.
\end{abstract}

Key words: perception, games, didactic resources, teaching, learning. 


\section{INTRODUCCIÓN}

La educación y su praxis han sufrido una serie de cambios tanto en sus formas de enseñanza como en sus contenidos y sus objetivos. Bajo esta dinámica de cambio también ha crecido la constante preocupación del profesor por hacer de su proceso de enseñanza y aprendizaje algo más duradero, efectivo y aplicable. Ya no basta con memorizar cierta cantidad de información, si no se logra identificar su utilidad tanto en la escuela como en la vida cotidiana; por ello ha comenzado a pensar de una manera distinta, tanto sus formas de enseńanza (didáctica) como los contenidos de la misma (conocimientos disciplinares), de manera tal que logre consolidar procesos educativos que se orienten más a la resolución de problemas y a la exploración de la creatividad que de un estado memorístico como se ha desarrollado frecuentemente en la escuela.

Esta preocupación ha llevado a los docentes a pensar cómo los jóvenes pueden adquirir elementos conceptuales que favorezcan el desarrollo de destrezas adecuadas para comprender, interpretar, actuar y transformar en sus propios contextos, ya que éstos están en constante cambio y, en esta medida, sus aprendizajes. En efecto, las prácticas educativas deben estar orientadas a "innovar tanto en los currículos como en los modelos didácticos, y entender al educando como un ser lleno de saberes que pueden ser articulados a nuevos conocimientos, y de esta manera, promover un espíritu crítico y reflexivo" (Melo, 2017, p. 927), teniendo en cuenta tanto los intereses como los conocimientos de los estudiantes y docentes, el contexto y los nuevos desafíos educativos.

El pensamiento que se tiene sobre las formas de los procesos de enseñanza-aprendizaje en la escuela son tan variados como sus recursos; en este sentido, las concepciones ${ }^{1}$ de docentes y estudiantes han sido permeadas por afirmaciones sociales como la subvaloración del juego y el jugar en todas las etapas de aprendizaje y campos del conocimiento, concibiendo esta actividad como un gasto de energía sin ningún sentido, o en los casos escolares, una actividad exclusiva

\footnotetext{
${ }^{1}$ En el contexto educativo, se entiende como concepción la idea que poseen las personas frente a los procesos de enseñanza y aprendizaje (Vilanova, Mateos y Basilisa, 2011).
} 
para los niños y niñas en los primeros años de vida, desvirtuando el valor del juego en la construcción del conocimiento, de la sociedad e incluso de la cultura. Al respecto, Huizinga (2000, p. 12) manifiesta que el juego es más viejo que la cultura y esta acción ha permitido que las personas construyan su cultura; no es simplemente un medio para el desgaste o para pasar el tiempo, ya que "en cuanto tal, traspasa los límites de la ocupación puramente biológica o física, es una función llena de sentido”.

Diversas investigaciones a lo largo de los últimos años han apuntado a considerar esta actividad un elemento importante en el desarrollo integral no sólo de los nińos, sino de los sujetos en cualquier etapa de su vida. Autores en distintos campos del conocimiento han realizado aportes de los cuales se extraen elementos importantes para entender la incidencia de éste en el desarrollo del hombre y de la sociedad. Desde el campo pedagógico se encuentra Fröbel (citado en Helmut, 1993), por ejemplo, que en sus estudios afirmó que el juego favorece el desarrollo cerebral y la formación del carácter, mientras que Groos (citado en Martínez y Villa, 2008) lo considera como un ejercicio preparatorio para la maduración y el desarrollo del pensamiento.

Desde otra perspectiva, autores como Sigmund Freud y Melanie Klein (mencionados en Raznoszczyk de Schejtman, s/f) pensaron que el juego es de origen biológico, considerado un efecto puramente físico y no un proceso evolutivo. En éste, el niño es llevado por sus deseos e impulsos; se trata de la posibilidad para liberar emociones reprimidas, para fantasear ante la imposibilidad de hacer de un pensamiento un hecho real, es una asociación entre lo que es real y lo imaginario. A través del juego el niño logra controlar los sucesos angustiosos de su día a día.

Por otro lado, Lev Vygotsky considera que el juego va más allá de una simple acción biológica, que produce placer y que es espontánea; éste identificó su valor como elemento integral primordial en el desarrollo cognitivo de los niños, y que posibilita la creación de la zona de desarrollo próximo, sin la cual no se podrían desarrollar procesos cognitivos superiores (Vygotsky, 2000), momento en el que se debe tener en cuenta tanto el contexto en el que se envuelve el niño o niña, como las representaciones que se generan con el 
juego y hecho de jugar. La zona de desarrollo próximo se refiere al proceso de construcción de conocimiento del niño y de interacción social en relación con su entorno, que se desarrolla con el juego, y se le atribuye que: "a partir de éste se adquiere el habla, la resolución de problemas en la interacción conjunta con un adulto, en las prácticas escolares" (Baquero, 2001, p. 139).

En este mismo sentido social, Morales (2013) menciona que el psicoanalista Donald Winnicott lo considera una actividad libertaria, que promueve la creatividad y la imaginación, y es capaz de distensionar y dar placer al que juega. En este sentido, se desarrollan procesos cognitivos complejos en la acción de jugar; conduce a la persona que juega a elaborar una serie de relaciones y asociaciones de lo que ya conoce con elementos nuevos en el que el otro desempeña un papel importante y no necesariamente es un par.

Frente a los procesos biológicos, se deben tener presentes los procesos cerebrales en la acción de jugar. Éstos van más allá de un impulso o un sentimiento, de un condicionamiento o una filiación. Gran parte del aprendizaje humano se produce en la corteza cerebral dependiendo de los estímulos que reciba, los cuales permiten desarrollar un sistema creativo en diferentes ambientes, incluso amenazantes; su estructura definida para los distintos procesos cognitivos permite entender la importancia de esta actividad en el desarrollo humano.

Cuando una persona juega, su cerebro produce una serie de sustancias como la dopamina, que actúa sobre el sistema nervioso y con la cual se fortalece la memoria corporal y muscular. Adicional a ello, durante el acto de jugar se produce serotonina, que regula la ansiedad y el estado de ánimo, y la acetilcolina, que promueve un estado de atención, aprendizaje y memoria. Jugando también se generan una serie de neurotransmisores como las encefalinas y las endorfinas, que se encargan de generar tranquilidad, bienestar y felicidad, estados propicios para desarrollar un ser creador e innovador; "es así como estos neurotransmisores producidos por el cerebro humano son básicos para la creación de nuevos seres fantásticos a nivel de la creatividad humana" (Jimenez, 2005, p. 113).

El juego en la etapa infantil es de gran significancia, pues a través de éste el niño desarrolla sus aprendizajes; sin embargo, se ha visto 
como una actividad exclusiva de los niños, desconociendo la naturaleza lúdica de los seres humanos. El juego va más allá de una función de crianzas o biológica; éste contiene un sinnúmero de significados para el que juega y esa condición le permite realizar procesos mentales que favorecen el desarrollo de la creatividad, la imaginación, la afectividad, la motricidad y la sociabilidad en cualquier etapa de la vida, ya que produce una transformación de sí mismo y del medio, al mismo tiempo que posee un gran potencial en los contextos educativos como

una actividad altamente potenciadora del aprendizaje de los seres humanos, de su evolución cognitiva, afectiva y social; es un proceso mediante el cual se construye y transforma con libertad y alegría, y se motiva al estudiante de manera autónoma, dinámica y creativa a realizar su propio proceso de aprendizaje (Melo y Barbosa, 2014, p. 61).

Las prácticas educativas que han desarrollado los autores durante sus prácticas en el aula han evidenciado que, por medio del juego, se aprende, y que es un elemento potenciador del desarrollo de esos aprendizajes; sin embargo, se debe tener en cuenta lo que piensan tanto docentes como estudiantes sobre el aporte de la inclusión de esta actividad en el contexto escolar. Para ello se empleó la encuesta como instrumento de recolección de datos, lo que permitió un acercamiento al pensamiento y la práctica del docente, al igual que a la del estudiante en su cotidianidad. En este sentido, se coincide con Marcelo (1987) cuando manifiesta que la investigación del pensamiento del docente logra un acercamiento a su desarrollo profesional, por ende, se puede hacer una comprensión de las conductas ante un determinado momento de enseñanza.

De hecho, las concepciones de los docentes tienen una fuerte influencia sobre lo que se enseña, cómo se enseña y cuál es el fin de esta enseñanza. Antiguamente, y aún en la actualidad, en algunos casos, se considera que los conocimientos son exclusivos de un área y son suficientes para el proceso de aprendizaje, desconociendo las diferentes relaciones que existen entre los distintos campos del saber y del contexto, lo que hace que se produzca un constructo difuso 
que limita la exploración y la creatividad tanto de la labor docente como del proceso de aprendizaje de los educandos.

Frente a la definición de "concepciones", se han encontrado diversas posturas en el contexto escolar que contribuyen a una lectura reflexiva sobre el tema del uso del juego en los contextos educativos. En las concepciones se reconocen muchos factores incidentes como las creencias y las vivencias individuales y colectivas que dan forma a los significados de los sujetos y se reconocen dos componentes: uno es lo que se concibe, lo otro es cómo se concibe, lo que permite conformar los marcos de referencia (Arbeláez, 2001).

También se entiende por concepciones a los "organizadores implícitos de los conceptos, de naturaleza esencialmente cognitiva y que incluyen creencias, significados, conceptos, proposiciones, reglas, imágenes mentales, preferencias, etc., que influyen en lo que se percibe y en los procesos de razonamiento que se realizan" (Azcárate, 2003, mencionado en Solbes y Torres, 2013, p. 64).

Frecuentemente, para el estudio de las concepciones de los docentes sobre la enseñanza y el aprendizaje se emplean modelos tanto teóricos como conceptuales de temas específicos. Se puede inferir que las concepciones tienen presente un juicio valorativo de lo que se cree, lo que se sabe y lo que se expresa de una realidad específica (Pozo y Scheuer, 2006).

Al tratar de comprender las concepciones de los docentes en los procesos de enseñanza y aprendizaje es conveniente identificar los puntos de partida para el análisis del mismo y, para este caso, se tendrán en cuenta tres variables:

- Son conocimientos explícitos que se manifiestan principalmente a través del lenguaje, bien sea oral, gráfico o escrito, entre otros.

- Son conocimientos enmarcados en contextos específicos; en éste se deben tener en cuenta la realidad y las creencias del docente, del estudiante y del entorno.

- Son un mismo fenómeno. Indica que se deben estudiar en conjunto ya que ninguno de los aspectos que inciden en los procesos de enseńanza y aprendizaje están desligados de otro y de esta manera se moldea el aprendizaje (Arancibia y Badia, 2015). 
Conocer las concepciones del docente, considerándolo un sujeto crítico y reflexivo, capaz de tomar decisiones racionales, permite comprender tanto sus posiciones como sus actitudes frente a su labor y profesión, ya que cada uno determina la manera de orientar sus prácticas, en el caso educativo del currículo, al tiempo que ofrece respuestas del mismo sin desligarse de la normatividad de cada institución ya que, aunque existe autonomía para los mecanismos de enseñanza, éstos deben desarrollarse bajo unos parámetros mínimos ya establecidos en las leyes educativas.

Estas concepciones permean de manera directa o indirecta el trabajo tanto del maestro como del estudiante, e inciden en cierta medida en el desarrollo del currículo, fundamentándose en su propio estilo de aprendizaje (Tobin y Mc Robbie, 1997). En el caso del docente, sus concepciones son consideradas lo suficientemente válidas para promover el pensamiento en sus educandos y para moldear, en cierta medida, su comportamiento. Éste enfatiza su práctica educativa en sus formas de pensar, pero no en las nuevas tendencias que se puedan generar de manera espontánea en el aula, presentando cierta resistencia al cambio.

En vista de la fuerte incidencia que adquiere la concepción docente en las formas y contenidos de las prácticas educativas, se puede determinar ésta como una variable con un fuerte componente cognitivo que permite reflexionar, y es derivado de las creencias y valores del mismo sujeto, lo que le aporta un sentido particular y único y le da sentido al desarrollo de las conductas tanto del que enseña, como del que recibe la enseńanza (Pozo y Scheuer, 2006).

De la misma manera que las concepciones del docente facilitan la comprensión de su quehacer, es importante conocer y comprender los pensamientos de los estudiantes frente a sus procesos de aprendizaje, debido a que todo lo que circunda al joven permea su pensamiento; es por ello que se coincide con Ovejero, Rodríguez, Becedoniz, Herrero, Gómez y Hernández (2005), quienes se refieren a que el rendimiento académico de los adolescentes está relacionado con la percepción que tiene del estilo educativo de sus maestros, de sus padres y de su clima familiar.

Estos hechos sugieren que los docentes deben comenzar a realizar una revisión autocrítica de sus prácticas pedagógicas, además de 
pensar en otro sentido los contenidos curriculares y de proyectos de las escuelas, ya que aún persisten prácticas tradicionales de educación que, en un gran porcentaje, se han convertido en procesos anquilosados y memorísticos; éstos son algunos de los aspectos que han desmotivado al estudiante hacia sus prácticas educativas, lo que Glasersfeld (1993) ha llamado una desilusión generalizada.

A partir de estos hechos, el estudiante elabora su propia concepción con base en toda la información que recibe en la escuela desde los contenidos vistos en clase, desde las relaciones sociales que se generan en la escuela y desde sus propios intereses y destrezas; de esta manera, él va elaborando juicios valorativos sobre sus aprendizajes y los mecanismos de enseñanza que ha empleado en lo que ha recorrido de su vida, bien sea en la escuela o en la vida cotidiana.

La motivación del estudiante tiene una fuerte influencia sobre su proceso de aprendizaje, lo mismo que el contexto sociocultural, los recursos con que se cuenta para el desarrollo educativo y los métodos empleados para llevar a cabo las actividades propias de la labor docente; adicional a ello, las exigencias actuales de la sociedad y sus cambios vertiginosos y frecuentes llevan a los docentes a establecer nuevas estrategias de enseńanza, con las cuales el estudiantes logre adquirir las competencias necesarias para desarrollarse y adaptarse a su contexto social.

Es así que la motivación del estudiante se convierte en un componente del proceso educativo que afecta la forma de pensar y de desarrollar su proceso de aprendizaje. Ya que la motivación es un factor que incide en los resultados de los procesos de aprendizaje, el docente debe orientar sus estrategias a generar este sentimiento en sus educandos para despertar su interés, provocar su curiosidad, establecer desafíos y, en fin, lograr una comprensión profunda y un conocimiento genuino.

Por estrategia de aprendizaje se puede hacer referencia a las secuencias de acciones dirigidas al logro de unas metas ya establecidas previamente (Stone, 1999), con las cuales se despierte el interés del educando, el gusto por lo que hace y aprende, así como su capacidad de comprensión de su realidad. Ello sugiere que el docente debe estar en constante innovación de su práctica educativa, en la que debe involucrar no sólo los recursos teóricos, sino elaborar sus propias herramientas de manera creativa. 


\section{METODOLOGÍA}

Realizar un acercamiento a las concepciones de docentes y estudiantes frente al juego fue posible empleando instrumentos de recolección de información de corte cualitativo, como la encuesta. Para la construcción de las encuestas como "uno de los elementos básicos en la realización de cualquier investigación social” (Hernández, 2001, p. 241) se determinaron objetivos y alcances, en los que se tuvo en cuenta las características socioeconómicas de la zona y la población con la cual se desarrollaba la investigación, además de los resultados académicos en las pruebas tanto institucionales como regionales, entre otros.

Estos cuestionarios constaban de 15 preguntas cada uno, unas de carácter abierto y otras de carácter cerrado. Cada una fue diseñada para identificar las diferentes percepciones del tema de investigación, la aplicación que se tenía del mismo y los modos o maneras de uso en diferentes contextos.

Respecto a los contenidos de las encuestas de los docentes, las preguntas estaban orientadas a conocer, por una parte, cuestiones propias de la práctica docente como la preparación del material usado en las clases, sus contenidos y actividades, modo evaluar, uso de herramientas, didácticas no convencionales, soporte epistemológico de sus diseńos metodológicos entre otros. Por otra parte, se indagó si tenían conocimiento epistemológico del tema en cuestión.

Posterior al diseño y estructuración de las encuestas, éstas fueron sometidas a revisión y validación por tres expertos en el tema del juego, los cuales realizaron sugerencias para mejorar el instrumento. Estos pares evaluadores fueron seleccionados de acuerdo con su trayectoria de investigación sobre el juego y sus aportes al desarrollo social integral de los sujetos. Para esta evaluación se diseñaron formatos que contenían ítems sustanciales para su aplicación y fueron enviados a los evaluadores. Al finalizar la revisión se realizó la reestructuración de las encuestas para aplicarlas a docentes y estudiantes.

Luego de haber realizado la revisión y reestructuración de los instrumentos se aplicaron las encuestas durante el segundo semestre de 2014 de la siguiente manera: 83 encuestas a docentes tanto de sexo masculino como femenino de todas las áreas de conocimiento 
de un total de 98 miembros de la institución educativa. En cuanto a los estudiantes, se realizaron 114 encuestas a niños y niñas en edades que oscilan entre los 11 y los 13 ańos, de un total de 140 pertenecientes a sexto grado de la jornada vespertina.

En cuanto a su aplicación, se realizó con los dos grupos una descripción de los objetivos de la encuesta y se les indicó que formaba parte de un trabajo de investigación educativa y se aclararon inquietudes que surgieron antes de comenzar con el diligenciamiento.

Para su proceso de tabulación se diseñaron categorías de análisis emergentes (que nacieron con el levantamiento de la información) basadas en los objetivos de investigación y en las respuestas que dieron los encuestados sobre su concepto de juego, las cuales se enmarcaron como conceptos sensibilizadores (Elliot, 1990). Estas categorías se diseñaron teniendo en cuenta que las encuestas eran de corte cualitativo; luego de ello, se procedió a realizar el análisis de las respuestas a cada pregunta.

\section{ANÁLISIS DE LOS RESULTADOS}

En este apartado se muestran los resultados de la aplicación de la encuesta y el análisis de los mismos; la información obtenida se cruzó con el marco teórico del trabajo investigativo, el cual hacía referencia al juego y a su incidencia en el aprendizaje desde la perspectiva filosófica, didáctica, pedagógica, psicológica y neuropedagógica; los antecedentes que mostraban el uso del juego como recurso en el ámbito educativo; el problema de investigación que nació de la necesidad de mejorar los resultados académicos de los estudiantes y los objetivos, con el fin de entablar una relación con cada uno de ellos para evidenciar la consistencia del proceso.

Se partió del análisis de cada pregunta de las encuestas realizadas a docentes y estudiantes (83 docentes y 114 estudiantes), cuestionarios que fueron validados por expertos en el tema de juego y didáctica. Para ello se crearon categorías de análisis por cada pregunta del cuestionario, debido a que se elaboró con preguntas abiertas. De acuerdo con las categorías emergentes, se realizó su respectiva tabulación y consolidación de resultados en términos cuantitativos. Estos resultados fueron analizados también en 
términos cualitativos. La interpretación y su respectivo análisis se orientaron bajo la mirada del problema de investigación, ya que esto permitió entender las ideas previas y los intereses tanto de docentes como de estudiantes.

\section{Encuesta aplicada a docentes}

Teniendo en cuenta que la encuesta se diseñó con preguntas abiertas para conocer la percepción de docentes y estudiantes respecto al juego, se presentan los resultados de las mismas en términos cuantitativos, lo que obedece a las categorías emergentes; luego de ello se hace el proceso cualitativo donde se registró el análisis del docente investigador de acuerdo con los conocimientos previos respecto del tema.

Sobre las respuestas relacionadas con la pregunta ¿cómo define usted el juego?, de acuerdo con las categorías de análisis que se generaron, se puede evidenciar que cerca de la mitad de los docentes encuestados considera que el juego es sólo una actividad que tiene como finalidad la diversión; también es una actividad lúdica que se emplea para desarrollar destrezas. En términos generales, se ve al juego como una actividad que transmite algo y que sirve para desarrollar alguna actividad social y humana (véase gráfica 1).

- GRÁFICA 1. Definición del juego de acuerdo a docentes encuestados, en porcentajes

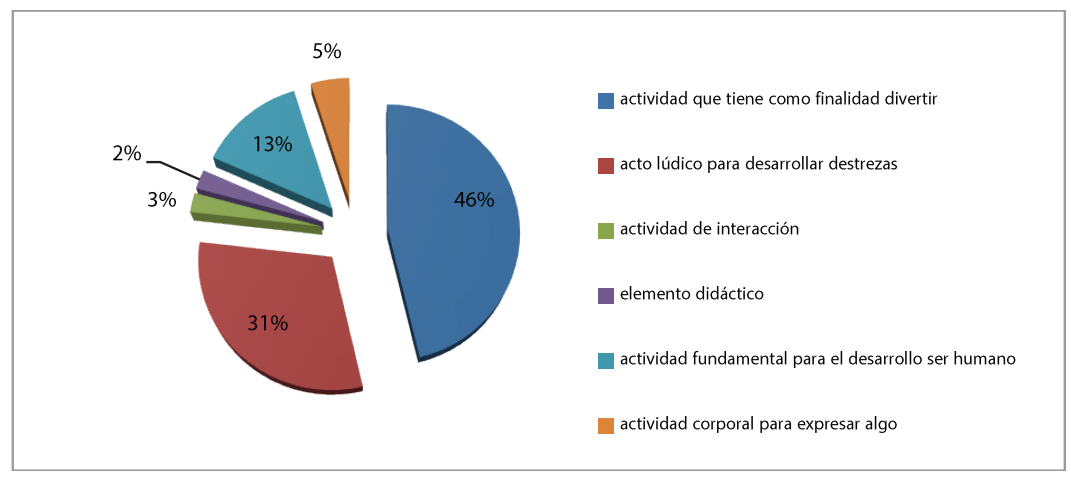

Fuente: elaboración propia.

En relación con el potencial desarrollador que puede generar el juego en niños y niñas, un gran porcentaje de los docentes (98\%) 
consideró que sí servía para promover el desarrollo y el aprendizaje en los niños; las personas que respondieron que tal vez (2\%), argumentaron que, dependiendo de la aplicación y la finalidad del juego, éste puede servir para promover el desarrollo de los educandos; de lo contrario, sólo serviría para divertirse.

La mayoría de los docentes conocen juegos de mesa y han tenido más acercamiento e interacción con éstos; también han practicado juegos tradicionales como el yo-yo, canicas, golosa, salto de soga, entre otros; debido al ambiente escolar, también se han practicado juegos deportivos, algunos se han empleado con las normas de juego establecidas y en otros casos se han hecho variaciones para ser ajustadas a un propósito pedagógico, entre otros. Esto demuestra que todos han tenido, de una u otra manera, un acercamiento al juego y esto les ha permitido entablar lazos sociales (véase gráfica 2).

- GRÁFICA 2. Tipos de juegos que conocen los docentes encuestados, en porcentajes

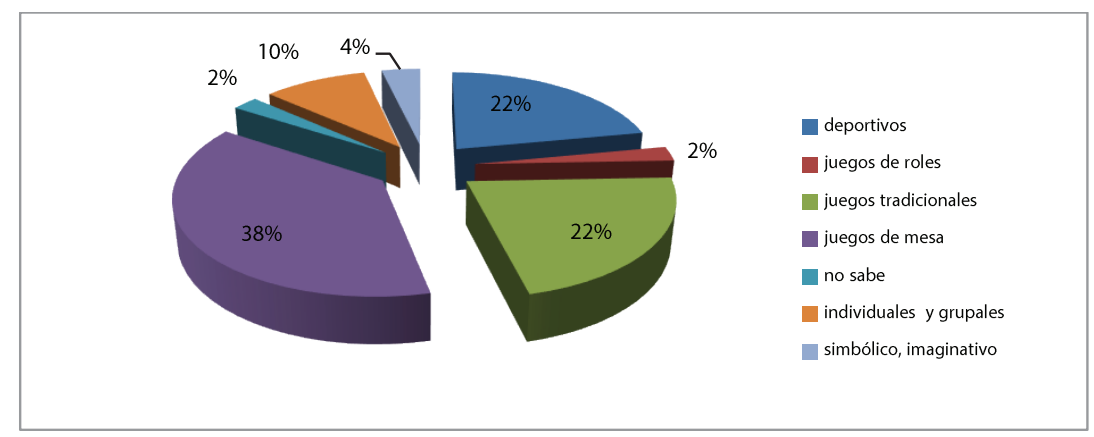

Fuente: elaboración propia.

Los docentes consideraron como primer elemento para clasificar los juegos las edades y los objetivos de los juegos. Juzgaron que los grupos deben delimitarse de acuerdo con su condición psicológica en términos de edad; también que los objetivos son clave a la hora de clasificarlos, ya que se puede hacer un juego para aprender o simplemente para pasar el tiempo. Por otro lado, expresaron que eran importantes las necesidades y los puntos de vista de los jugadores. Muy pocos valoraron que se podía tener en cuenta el desarrollo cognitivo que se hace presente a la hora de jugar (véase gráfica 3). 
GRÁFICA 3. Criterios que utilzarían los docentes encuestados para clasificar los juegos, en porcentajes

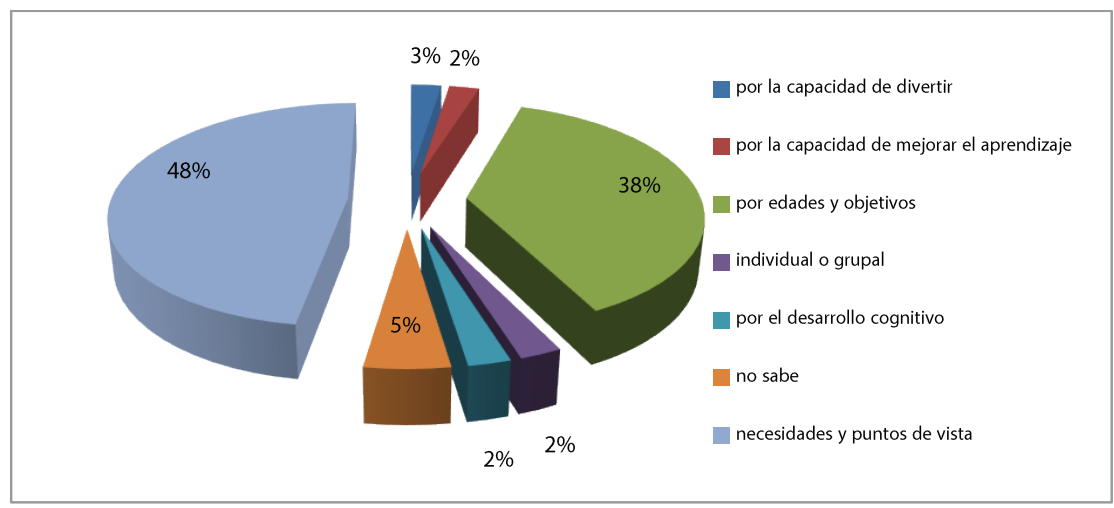

Fuente: elaboración propia.

Es evidente que, aunque la mayoría de los encuestados han empleado metodologías lúdicas para sus prácticas docentes en algún momento, otros no lo han considerado un elemento pedagógico. Entre las prácticas lúdicas que se han empelado se encuentran los juegos de roles, de motivación, juegos de mesa, de escritura y deportivos (véase gráfica 4).

- GRÁFICA 4. Docentes encuestados que han usado o no metodologías lúdicas en su práctica, en porcentajes

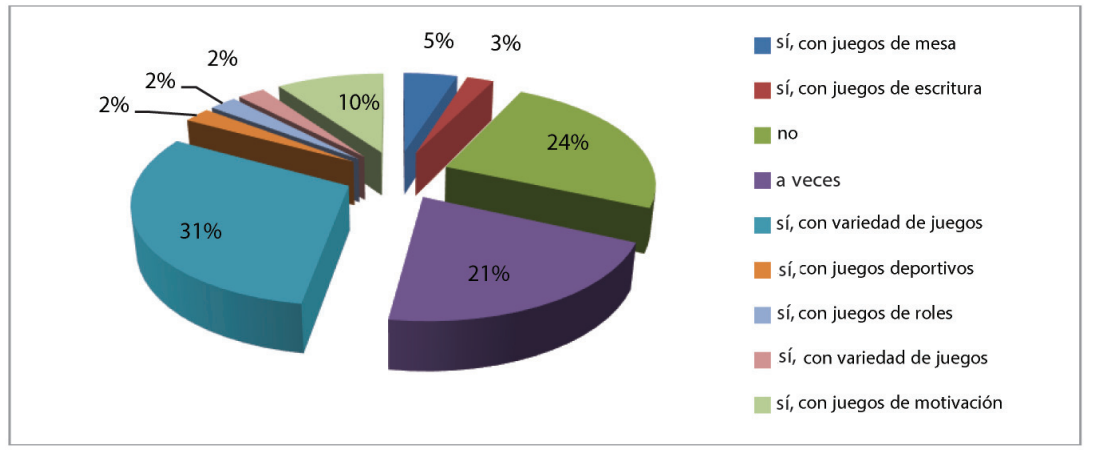

Fuente: elaboración propia.

Si bien es cierto que el juego se emplea en los primeros años de vida, no debemos olvidar que todos los seres humanos somos lúdicos por naturaleza; es por ello que los docentes sí han participado 
de juegos con sus estudiantes por medio de videojuegos, en las prácticas deportivas o en las dinámicas grupales que se desarrollan en clase. El $17 \%$ de los docentes encuestados mencionaron que no han jugado con sus estudiantes debido a que no les parece una actividad de importancia (véase gráfica 5).

- GRÁFICA 5. Docentes encuestados que han jugado o no con sus alumnos, en porcentajes

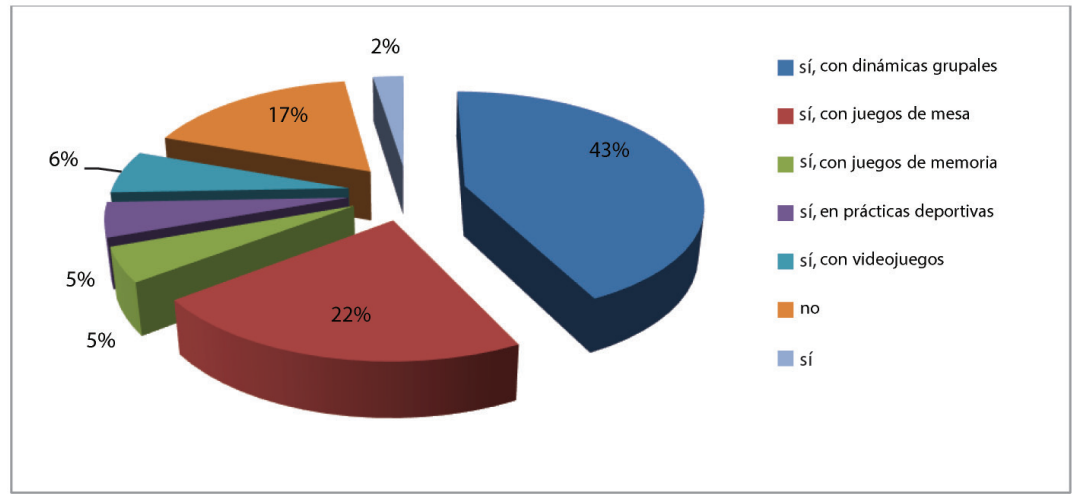

Fuente: elaboración propia.

Ante la pregunta de si consideraban posible desarrollar una estrategia pedagógica cuando se juega en la escuela, 94\% de los docentes respondió que sí es posible y viable, además de considerarlo una actividad altamente motivadora para los estudiantes; el restante $6 \%$ considera que no es posible debido a la falta de recursos y de motivación por parte de la administración.

De acuerdo con las respuestas de los docentes, se identificó que $78 \%$ en algún momento ha utilizado el juego como estrategia pedagógica; en la mayoría de los casos emplearon actividades como los juegos de concurso, juegos de memoria y juegos de destreza. El 22\% restante no ha empleado esta clase de estrategias porque considera que no permiten un nivel de organización ni concentración a la hora de realizar una actividad de clase.

El 95\% de los docentes encuestados consideró que el juego como estrategia didáctica sí podía favorecer los aprendizajes de los estudiantes desde diferentes miradas; por un lado, mencionaron que fortalecía la confianza y el interés por el estudio, también lo consideraron como un fuerte dinamizador y facilitador, además que sí 
perciben que puede mejorar el proceso cognitivo. El restante 5\% manifestó que no sabía si era posible emplear esta actividad en sus planes de aula (véase gráfica 6).

GRÁFICA 6. Opinión de los docentes encuestados sobre el juego como estrategia para favorecer el aprendizaje de sus alumnos, en porcentajes

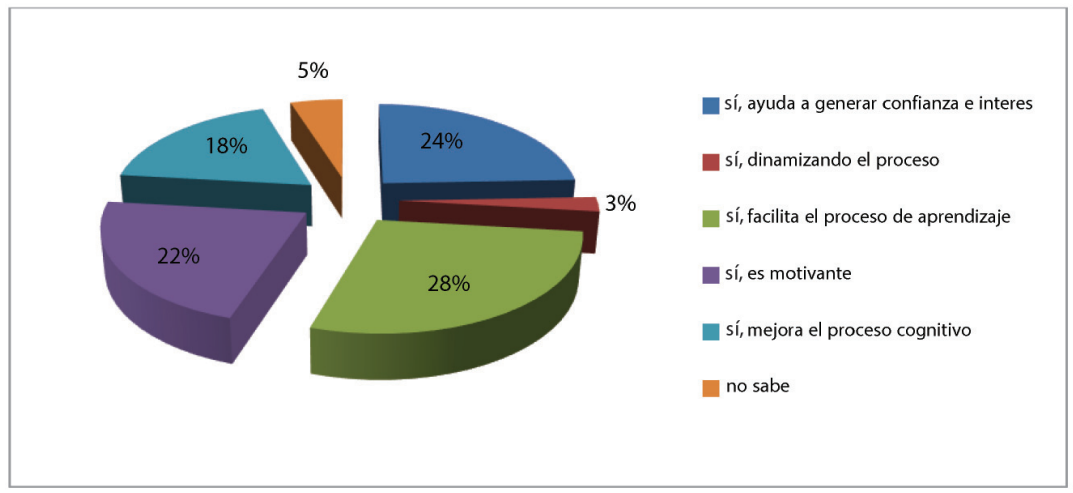

Fuente: elaboración propia.

\section{Encuesta aplicada a estudiantes}

Para el análisis de los resultados de las encuestas a estudiantes se pretendía obtener datos de los conocimientos previos de los educandos para, posteriormente, abordar el tema del juego en el aprendizaje escolar. Al igual que con los docentes, se tuvo en cuenta categorías de análisis y se presentan los resultados en términos cuantitativos y cualitativos. A continuación, se procede a mostrarlos.

Para $62 \%$ de los estudiantes, el juego es diversión. Ven en él la posibilidad de ser alegres, espontáneos y creativos. Por otro lado, lo ven como la posibilidad de compartir con otras personas dentro y fuera de la institución, es entretencion (véase gráfica 7).

Al preguntar a los estudiantes por qué consideran algo como un juego, las respuestas tienen relación directa con las respuestas a la pregunta anterior. Ellos ven que, a partir del juego, se pueden divertir y compartir con el otro, aspecto que facilita su desarrollo social y humano. Para ellos jugar es una actividad del recreo y de las clases de educación física; sin embargo, la posibilidad de jugar se da en cualquier momento y lugar (véase gráfica 8). 
GRÁFICA 7. Concepción del juego según los estudiantes encuestados, en porcentajes

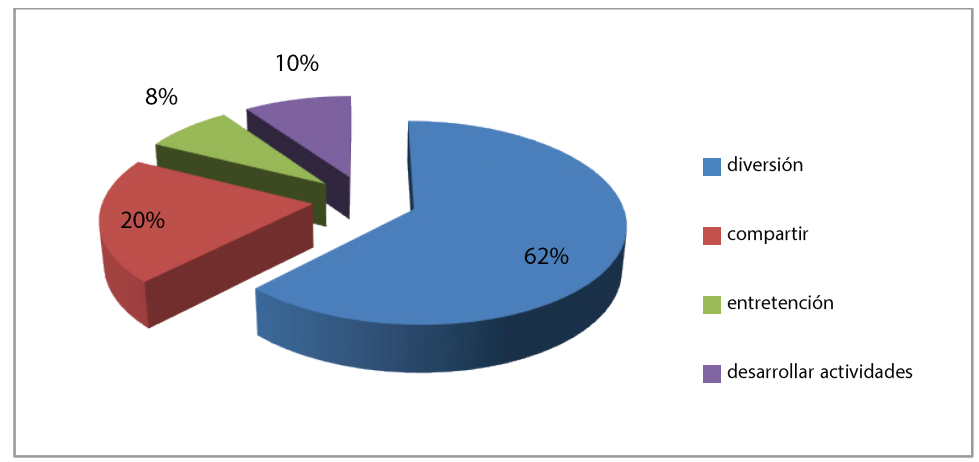

Fuente: elaboración propia.

- GRÁFICA 8. Razones de los estudiantes encuestados para considerar algo como un juego, en porcentajes

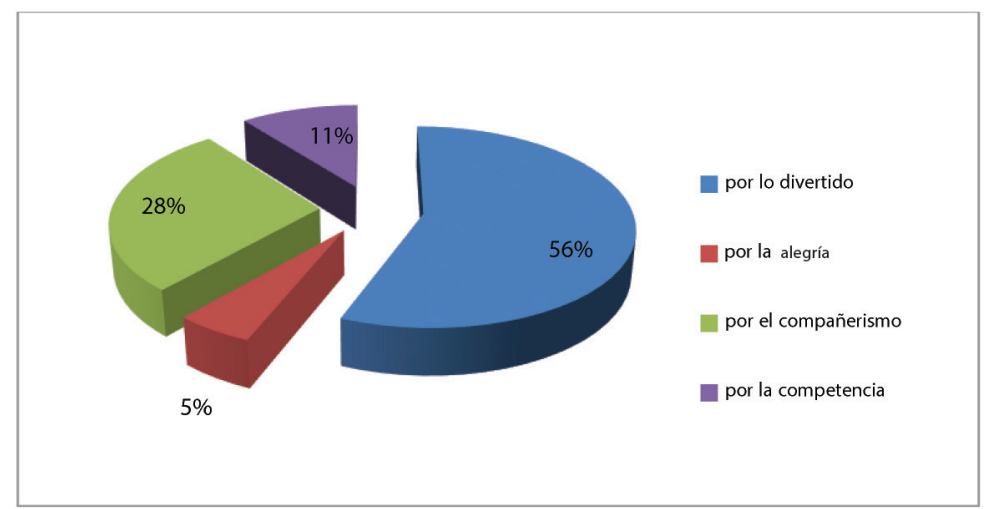

Fuente: elaboración propia.

Entre los estudiantes encuestados, 54\% mencionó que juega entre dos y tres horas diarias en diferentes espacios y momentos, el $46 \%$ restante juega de una a dos horas debido a las múltiples actividades que se les presentan en el contexto social en el que se desenvuelven. Esto evidencia que es una actividad de gran interés para los educandos ya que le dedican buena parte de su tiempo.

Debido a las actividades cotidianas de los niños se pudo determinar que los encuestados siempre están practicado juegos deportivos; de forma adicional a éstos, han practicado juegos tradicionales como la golosa, el yermis, el parque, el dominó, seguido de los 
juegos recreativos o que ellos mismos inventan y los videojuegos. Esto evidencia que el juego es una actividad que tiene un papel importante en la vida de los estudiantes, ya que siempre están jugando (véase gráfica 9).

- GRÁFICA 9. Clases de juegos que practican los estudiantes encuestados, en porcentajes

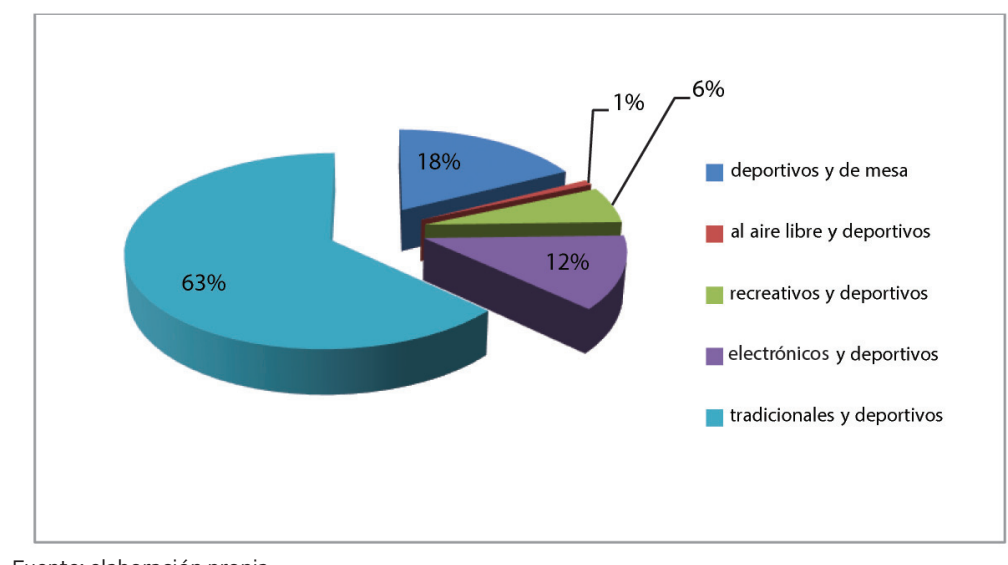

Fuente: elaboración propia.

Las respuestas de los estudiantes apuntan a que todos han jugado en compañía: 74\% manifestó que siempre juega con alguien; esto se debe a que siempre buscan a sus pares para hacer actividades de su interés como el ir al parque. El restante 26\% mencionó que jugaba en compañía o solos. De acuerdo con las respuestas se constató que, aunque ellos siempre juegan en compañía, hay ocasiones que lo hacen solos por múltiples motivos como el no querer estar acompańado o porque se encuentra solo en el momento de querer jugar; sin embargo, siempre emplean algún elemento para su actividad o tienen un amigo imaginario.

De acuerdo con lo mencionado por los estudiantes, ellos emplean cualquier elemento que puede ser útil para su juego, por ejemplo, platones, cobijas, cajas, etc. Adicional a ello se evidencia que, en su gran mayoría, usan elementos deportivos, especialmente balones y muy pocos tienen acceso a juegos electrónicos (véase gráfica 10).

Los niños y nińas encuestados ven en todos los lugares la posibilidad de jugar y de realizar sus actividades. Entre los lugares más 
recurrentes fueron el colegio, debido a que tienen a los amigos con los cuales juegan; la calle, ya que tienen amigos en su barrio y la casa, ya que la mayoría de ellos tienen hermanos y familiares que son cercanos en edades (véase gráfica 11).

- GRÁFICA 10. Clase de elementos o materiales con los que juegan los estudiantes encuestados, en porcentajes

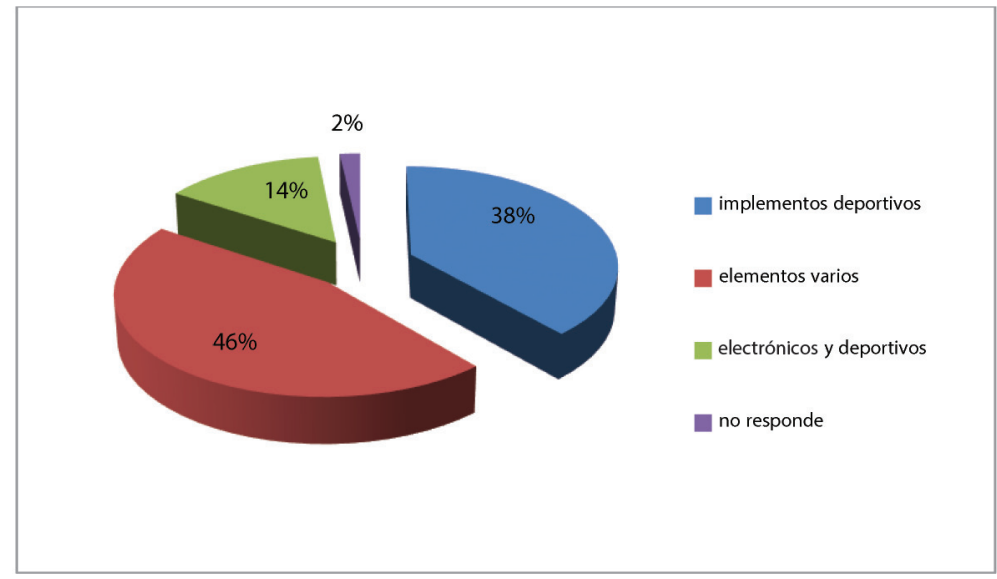

Fuente: elaboración propia.

- GRÁFICA 11. Lugares donde juegan los estudiantes encuestados, en porcentajes

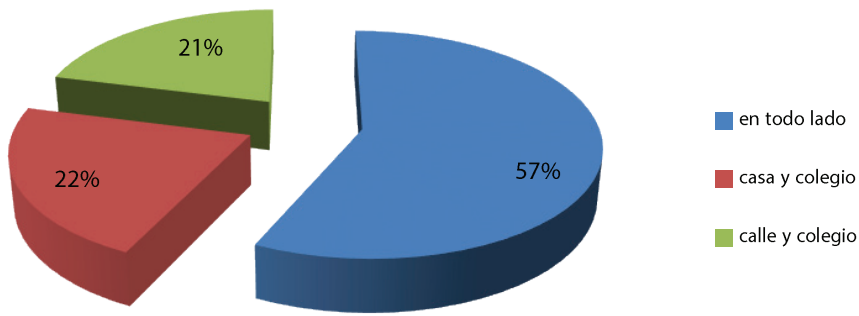

Fuente: elaboración propia. 
Debido al carácter motivador del juego, $72 \%$ de los encuestados consideró que sí se podía aprender jugando y mencionó algunas actividades que han realizado en el colegio con las cuales han aprendido algo. El 28\% restante consideró que no se aprende porque es una actividad sólo para la diversión.

Si bien para todos los niños el juego es una actividad importante en su vida, es también valioso destacar los motivos por los cuales lo consideran así. Las respuestas fueron diversas; sin embargo, se puede evidenciar que $42 \%$ lo ve importante porque se divierte, seguido del ejercicio físico y del aprendizaje, que representa 19\% (véase gráfica 12).

GRÁFICA 12. Razones por las que el juego es importante según los estudiantes encuestados, en porcentajes

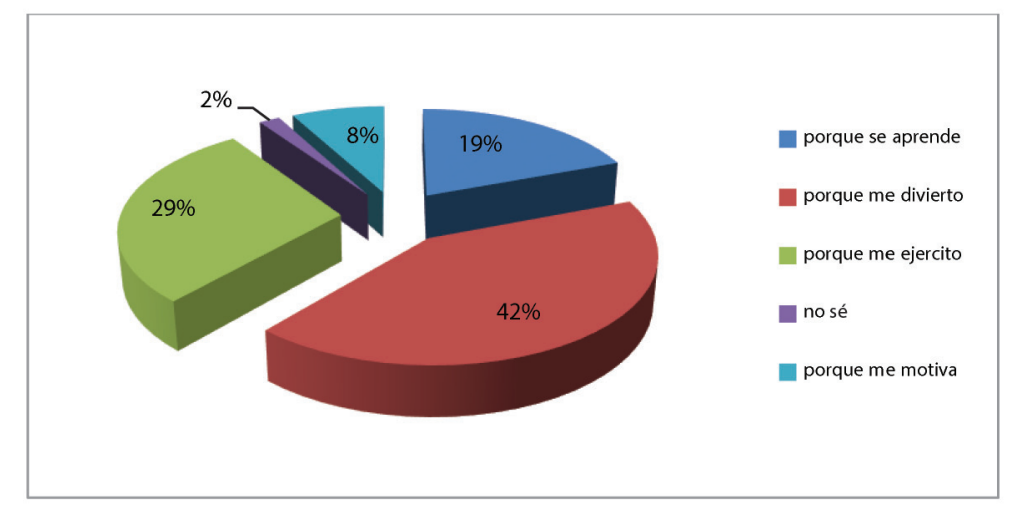

Fuente: elaboración propia.

Al finalizar el ejercicio de análisis de cada una de las preguntas, se procedió a realizar un cuadro comparativo donde se expone, en términos generales, las percepciones de docentes y estudiantes, a fin de encontrar afinidades y diferencias para comprender de mejor manera cómo se iba a abordar la construcción de juegos en la clase de ciencias, descrito en el cuadro 1. 
CUADRO 1. Paralelo entre las concepciones de docentes y estudiantes con respecto al juego

\begin{tabular}{|c|c|}
\hline & Análisis de las concepciones de los estudiantes \\
\hline $\begin{array}{l}\text { De acuerdo con los resultados, se puede determinar } \\
\text { que, en su mayoría, los docentes consideran } \\
\text { que el juego es una actividad lúdica que tiene } \\
\text { como finalidad divertir y que, de acuerdo con los } \\
\text { intereses y necesidades tanto del docente como } \\
\text { de la institución, puede favorecer el desarrollo } \\
\text { de los niños desde los aspectos físico, social y } \\
\text { emocional. Los docentes también destacaron en } \\
\text { el juego la capacidad de disfrutar y recrear no sólo } \\
\text { al que juega, sino a los espectadores también; } \\
\text { consideraron que las edades de los jugadores y los } \\
\text { objetivos de la actividad podrín ser elementos para } \\
\text { clasificar al juego. Otro aspecto que se identificó } \\
\text { es que cerca de la mitad de los docentes han } \\
\text { empleado juegos de mesa, de roles, deportivos, y } \\
\text { han participado en los juegos con sus estudiantes } \\
\text { en dinámicas de grupo que han sido diseñadas } \\
\text { previamente para ello, ya que ven en el juego } \\
\text { una posibilidad de ser usado como herramienta } \\
\text { para favorecer el aprendizaje. Los profesores de la } \\
\text { institución educativa consideraron que sí es posible } \\
\text { desarrollar una estrategia pedagógica a partir del } \\
\text { juego y que éste puede favorecer aprendizajes en } \\
\text { los estudiantes que juegan debido a que es una } \\
\text { actividad altamente motivante, que logra dinamizar } \\
\text { procesos y ayuda a generar confianza e interés en } \\
\text { el educando, haciendo que su aprendizaje cobre un } \\
\text { sentido y un significado. }\end{array}$ & $\begin{array}{l}\text { Los estudiantes de la institución educativa ven } \\
\text { al juego como un mecanismo para divertirse, } \\
\text { entretenerse y desarrollar actividades con sus } \\
\text { compañeros, amigos y familiares. Consideran que el } \\
\text { juego los hace reír y los divierte, y manifiestan que } \\
\text { las actividades que desarrollan son agradables por } \\
\text { lo divertido de las mismas y, en su gran mayoría, } \\
\text { juegan de dos a tres horas diarias. La mayor parte } \\
\text { de los estudiantes encuestados conoce y ha tenido } \\
\text { contacto con juegos tradicionales como la rana, } \\
\text { el trompo, las canicas; también han desarrollado } \\
\text { juegos deportivos como futbolito, mini-baloncesto } \\
\text { y voleibol, seguidos de juegos de mesa como el } \\
\text { parque, el dominó, las damas chinas, la escalera, el } \\
\text { bingo, entre otros; en lo que respecta a los juegos } \\
\text { electrónicos, los han usado en un menor porcentaje } \\
\text { debido al difícil acceso a éstos. Manifiestan que } \\
\text { siempre han jugado en compañía, bien sea con } \\
\text { amigos, compañeros de escuela o familiares; muy } \\
\text { pocas veces lo hacen solos, aunque en algunos casos } \\
\text { emplean personajes imaginarios. Para jugar usan } \\
\text { cualquier elemento que encuentren conveniente } \\
\text { para desarrollar su actividad como tapas de ollas que } \\
\text { usan como escudos protectores, cobijas que sirven } \\
\text { de capas de superhéroes, vasos plásticos que se } \\
\text { convierten en carros, aviones o barcos, entre otros. } \\
\text { actividad a la que ven como un motivante para ser } \\
\text { empleado durante las clases. } \\
\text { colegio, la casa y el parque o la calle. Por otro lado, } \\
\text { las percepciones de los estudiantes se encuentran } \\
\text { jivididas al preguntarles si consideran si aprenden } \\
\text { jua que unos consideran que sí es posible, } \\
\text { jue otros piensan que esta actividad es } \\
\text { jivertirse; sin embargo, piensan }\end{array}$ \\
\hline
\end{tabular}

\title{
CONCLUSIONES
}

\author{
Si bien es cierto que el juego ha sido percibido social y culturalmen- \\ te como una actividad para niños que cumple sólo el objetivo de \\ distraer, alegrar, o sólo para pasar el tiempo, en los contextos esco-
}


lares se ha reconocido su potencial en cuanto a mejorar los procesos de enseñanza-aprendizaje, evidenciando su aporte en los resultados. Sin lugar a duda, el ambiente en el que cualquier sujeto se desarrolla incide en su manera de pensar, de sentir y de proyectarse en el mundo. Es por ello que las percepciones de las personas son fuertemente influidas por el medio social, y de esta manera, se comienzan a moldear acciones, en este caso, las formas de enseñar y de aprender; de ahí la importancia de resaltar al juego como un fuerte recurso didáctico que favorece la obtención de los objetivos de la escuela, no sólo en resultados académicos, sino también en los resultados sociales, ya que permite mejorar las relaciones entre la comunidad.

Estas percepciones permean, en cierta medida, las prácticas de enseñanza y aprendizaje en ambientes escolares, y estos aprendizajes inciden tanto en el desarrollo de las relaciones interpersonales como del pensamiento, haciendo que se evidencie en cierta medida si las prácticas educativas de las instituciones educativas están orientándose a lograr aprendizajes que favorezcan la vida en sociedad, haciendo personas críticas, reflexivas, que aporten a la construcción de su sociedad.

Los resultados de la encuesta permitieron evidenciar que los docentes tienen una percepción más coloquial que conceptual del juego, de que orienta las diferentes áreas de conocimiento en la escuela, por ello en el mismo ámbito educativo se ha llegado a desvirtuar su potencial en lo pedagógico, en lo didáctico y en el desarrollo del ser humano en sus diferentes manifestaciones.

La encuesta también permitió realizar un acercamiento a las percepciones e intereses que tienen tanto docentes como estudiantes con respecto al juego y todos los elementos que lo componen con el fin de comprender las ideas que se tenían del tema y dio paso a determinar de qué manera se podía emplear el juego en los procesos educativos.

Los datos mostraron que todos los encuestados han tenido un acercamiento a algún tipo de juego y que lo identifican con facilidad, ya que lo han empleado en algún momento de sus vidas, bien sea en ambientes laborales, como es el caso de los docentes, o en ambientes sociales, como por ejemplo en la consecución de actividades con amigos o familiares, lo que indica que el juego está presente de alguna manera en todos los aspectos del ser humano sin importar su condición o nivel de madurez. 
En lo que respecta a los docentes, se puede observar que un bajo porcentaje considera que el juego puede favorecer sus prácticas educativas, pero al mismo tiempo piensan que esta actividad facilitaría los aprendizajes de los estudiantes durante el desarrollo de sus clases. Si bien los docentes han usado de una u otra manera recursos que llevan a prácticas de carácter lúdico, lo han empleado de manera esporádica debido a la alta exigencia que una actividad lúdica demanda; si aún no se dimensiona su carácter potenciador de aprendizaje y de desarrollo humano, tal vez se deba al desconocimiento conceptual y epistemológico del juego, o a las exigencias del currículo y la escuela.

Realizar un acercamiento a las percepciones y a los significados de docentes y estudiantes permite comprender ciertos comportamientos y dilemas que se presentan en las prácticas de aula; de ahí la importancia de conocer ideas previas de los educandos como punto de partida de la enseñanza para, de esta manera, diseñar propuestas didácticas encaminadas a ser modificadas constantemente de acuerdo con los contextos, los intereses y el propósito educativo.

Otro aspecto que incide y puede favorecer o debilitar un proceso de aprendizaje es lo que piensa el estudiante tanto de su orientador o maestro, como de su práctica educativa. Las percepciones de los estudiantes contribuyen al buen desarrollo de una temática de clase desde su conocimiento y comportamiento, también generan aportes que enriquecen la práctica educativa.

En efecto, los procesos de enseñanza y aprendizaje deben ser integrales, así como los sujetos; en éstos no sólo se hacen presentes los contenidos de un área, también se deben tener en cuenta la relación con el mundo de los valores, los principios de la convivencia, los socioafectivos, la motivación como factor fundamental, entre otros; por ejemplo, una persona con una fortaleza afectiva tendrá mayor predisposición al trabajo que una persona que tiene falencias en ese ámbito.

En este sentido, el docente debe asumir su rol de orientador y motivador de tal manera que contribuya a un buen proceso de aprendizaje, empleando recursos didácticos llamativos, innovadores, que además de fomentar los procesos cognitivos, sean capaces de fortalecer los aspectos socioafectivos que posibiliten generar una red de intercambios y la construcción de comunidad. 


\section{SUGERENCIAS}

Frente al proceso metodológico es importante tener en cuenta que, al ser una investigación de corte cualitativo, el investigador se enfrenta a respuestas subjetivas que pueden dar un rumbo distinto al proceso, por ello, es importante tener en cuenta y analizar todos los hallazgos del proceso.

Respecto al diseño de las encuestas, aunque fueron validadas por pares expertos, hubo algunas dificultades en las respuestas de los estudiantes, ya que consideraban que se debía responder para agradar al investigador. Se debe pensar en un mecanismo de ejecución que permita realizar el proceso de acuerdo con los objetivos planteados.

En cuanto al resultado de las encuestas, se evidenció que, en algún momento, los profesores han usado el juego como recurso didáctico para promover aprendizajes en sus estudiantes, sin embargo, es difícil para ellos articular de una manera permanente estos mecanismos, posiblemente por las concepciones que se tienen del juego. Se podría hacer un proceso de reflexión sobre el valor educativo del juego y el jugar en el gremio docente de Colombia, resaltando el potencial educativo al implementar recursos que sean de mayor acogida por los estudiantes.

\section{REFERENCIAS BIBLIOGRÁFICAS}

Arancibia, M. M., y Badia, A. (2015). Concepciones de profesores de secundaria sobre enseñar y aprender historia con Tic. Revista electrónica de investigación educativa. Redie, 17(2), 62-76.

Arbeláez, R. (2001). En el reconocimiento de las concepciones docentes se encuentra el camino del mejoramiento contínuo de la calidad docente. Revista Docencia Universitaria, 2(2), 1-11.

Baquero, R. (2001). Vigotsky y el aprendizaje escolar. Buenos Aires: Aique. Elliot, J. (1990). La investigación acción en educación. Madrid: Morata.

Glasersfeld, E. (1993). Constructivismo: el paradigma, el aprendizaje, la enseñanza y el cambio conceptual. Santiago: Universidad Santiago de Cali.

Helmut, H. (1993). Friedrich Fröbel (1872-1852). Perspectivas: Revista trimestral de educación comparada, XXIII(3-4), 501-519.

Hernández, B. (2001). Técnicas estadísticas de investigación social. Madrid: Diaz de Santos. 
Hiuzinga, J. (2000). Homo Ludens. España: Alianza/Emecé.

Jiménez, C. (2005). La inteligencia lúdica: juegos y neuropedagogía en tiempos de transformación. Bogotá: Aula Abierta Magisterio.

Marcelo, C. (1987). El pensamiento del profesor. Barcelona: Ceac.

Martínez, E., y Villa, S. (2008). El juego como escuela de vida. Karl Groos. Magister: Revista miscelánea de investigación, (22), 7-22.

Melo, M. (2017). Juguemos a respirar. Cómo entender el concepto de respiración desde el juego de "ponchados" en las clases de educación física. Bio-grafía, Escritos sobre la biología y su enseñanza, (Número Extraordinario), 926-932. Doi: https://doi.org/10.17227/bio-grafia. extra2017-7257

Melo, M., y Barbosa, R. (2014). El juego y sus posibilidades en la enseñanza de las ciencias naturales. Innovación Educativa, 14(66), 41-63.

Morales, L. (2013). El impulso lúdico: esencia y estructura del juego. Tonos digitales, (25), 1-27. Recuperado de https://dialnet.unirioja.es/servlet/articulo?codigo $=4494845$

Ovejero, A., Rodríguez, F., Becedoniz, C., Herrero, F., Gómez, P., y Hernández, E. (2005). La convivencia sin violencia. Madrid: MAD.

Pozo, J., y Scheuer, M. (2006). Nuevas formas de pensar la enseñanza y el aprendizaje. Barcelona: Grao.

Raznoszczyk, C. (s.f.). Los juegos del niño en la actualidad. Buenos Aires, Argentina: Universidad de Buenos Aires. Recuperado de https://www.psi. uba.ar/academica/carrerasdegrado/psicologia/sitios_catedras/obligatorias/054_ninez2/material/juegos_del_ni\%F1o_en_la_actualidad.doc

Solbes, J., y Torres, N. (2013). ¿Cuáles son las concepciones de los docentes de ciencias en formación y en ejercicio sobre el pensamiento crítico? Tecné, episteme y didaxis: revista de la Facultad de Ciencia y Tecnología (TED), (33), 61-85.

Stone, M. (1999). Enseñanza para la comprensión, vinculación entre la investigación y la práctica. Barcelona: Paidós.

Tobin, K., y Mc Robbie, C. (1997). Beliefs about the nature of science and the enacted science curriculum. Science and education, 6(4), 355-371.

Vilanova, S., Mateos, M., y Basilisa, M. (2011). Las concepciones sobre la enseñanza y el aprendizaje en docentes universitarios de ciencias. Revista Iberoamericana de Educación Superior, II(3), 53-75.

Vygotsky, L. (2000). El desarrollo de los procesos psicológicos superiores. Barcelona: Crítica. 